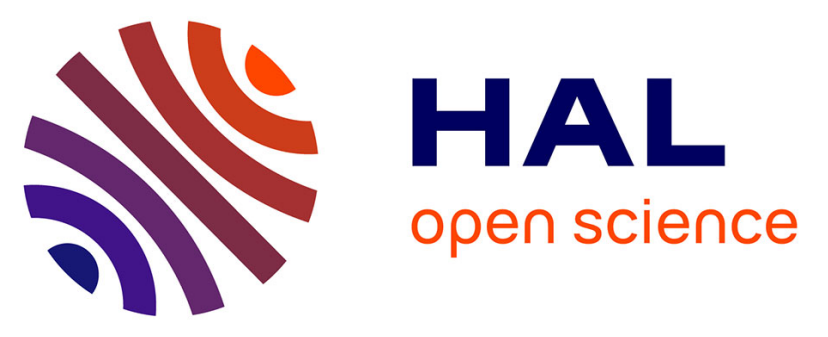

\title{
Functional dedifferentiation and reduced task-related deactivations underlie the age-related decline of prospective memory
}

\author{
Julie Gonneaud, Grégory Lecouvey, Mathilde Groussard, Malo Gaubert, \\ Brigitte Landeau, Florence Mézenge, Vincent de La Sayette, Francis Eustache, \\ Béatrice Desgranges, Géraldine Géraldine Rauchs
}

\section{To cite this version:}

Julie Gonneaud, Grégory Lecouvey, Mathilde Groussard, Malo Gaubert, Brigitte Landeau, et al.. Functional dedifferentiation and reduced task-related deactivations underlie the age-related decline of prospective memory. Brain imaging and behavior (Brain Imaging Behav), 2016, Epub ahead of print. 10.1007/s11682-016-9661-z . inserm-01417543

\section{HAL Id: inserm-01417543 https://www.hal.inserm.fr/inserm-01417543}

Submitted on 15 Dec 2016

HAL is a multi-disciplinary open access archive for the deposit and dissemination of scientific research documents, whether they are published or not. The documents may come from teaching and research institutions in France or abroad, or from public or private research centers.
L'archive ouverte pluridisciplinaire HAL, est destinée au dépôt et à la diffusion de documents scientifiques de niveau recherche, publiés ou non, émanant des établissements d'enseignement et de recherche français ou étrangers, des laboratoires publics ou privés. 
Functional dedifferentiation and reduced task-related deactivations underlie the agerelated decline of prospective memory.

Julie Gonneaud ${ }^{1,2,3,4}$, Grégory Lecouvey ${ }^{1,2,3,4}$, Mathilde Groussard ${ }^{1,2,3,4}$, Malo Gaubert ${ }^{1,2,3,4}$, Brigitte Landeau $^{1,2,3,4}$, Florence Mézenge ${ }^{1,2,3,4}$, Vincent de La Sayette ${ }^{1,2,3,5}$, Francis Eustache ${ }^{1,2,3,4}$, Béatrice Desgranges ${ }^{1,2,3,4} \&$ Géraldine Rauchs ${ }^{1,2,3,4}$

${ }^{1}$ INSERM, U1077, Caen, France

${ }^{2}$ Université de Caen Normandie, UMR-S1077, Caen, France

${ }^{3}$ Ecole Pratique des Hautes Etudes, UMR-S1077, Caen, France

${ }^{4}$ CHU de Caen, U1077, Caen, France

${ }^{5}$ CHU de Caen, Service de Neurologie, Caen France

Corresponding author: Géraldine Rauchs, $\mathrm{PhD}$

Inserm U1077, GIP Cyceron,

Boulevard H. Becquerel, BP 5229

14074 Caen Cedex, France.

Phone: + 33231470134 / Fax: + 33231470106

e-mail: rauchs@cyceron.fr 


\begin{abstract}
Prospective memory (PM) refers to the ability to remember to execute an intention at the appropriate moment in the future, which can be performed either at the appearance of an event (event-based, EBPM) or after a certain amount of time (time-based, TBPM). PM is generally impaired during aging but the cerebral substrates of this decline have been little investigated. Using functional Magnetic Resonance Imaging (fMRI), we investigated the neural bases of PM in 20 young and 20 healthy older adults. They were proposed a task of semantic categorisation of pictures (ongoing task). For some blocks, participants only had to perform this ongoing task while, for others, a PM instruction was added. In this case, a supplementary answer in response to a specific colour of border for EBPM or at specific time intervals for TBPM was expected. PM, and more particularly TBPM, declined in older adults. For both PM conditions, older adults recruited additional brain areas, but also showed reduced deactivations of other regions. These results are discussed in light of models of the aging brain.
\end{abstract}

Keywords: Prospective Memory, functional MRI, Healthy Aging, Event-based, Time-based 


\section{Introduction}

Prospective Memory (PM) refers to the ability to remember to execute an intention at the appropriate point in the future (McDaniel and Einstein 2000). Retrieval of an intention could be triggered by the appearance of an event, called event-based PM (EBPM), or after a certain amount of time has elapsed, called time-based PM (TBPM; Einstein and McDaniel 1990). Retrieval in PM is thought to rely on automatic process (i.e. spontaneous retrieval) when the prospective cue is salient or focal, when the ongoing task is little demanding, or when the PM cue and the intention are strongly related (Guynn et al. 1998; McDaniel and Einstein 2000). In all other situations (prospective cue not salient or non-focal, highly demanding ongoing task, prospective cue and intention weakly or not related), PM may rather rely on controlled processes such as strategic monitoring. Studies assessing the effect of age on PM report discrepant results (Henry et al. 2004; McDaniel and Einstein 2011 for reviews) that may be attributed to the differential involvement of automatic versus controlled processes according to the tasks. Thus, the age-related PM decline may be more pronounced in conditions in which controlled processes are particularly required (Craik 1986). Interestingly, TBPM may be more affected by aging than EBPM (Craik 1986; Einstein et al. 1995; Park et al. 1997). The greater reliance on self-initiated processes and difficulties for older adults to strategically monitor the time have been proposed to account for this greater decline in TBPM (Einstein et al. 1995; Mäntylä et al. 2009). Interestingly, the age-related variability in PM performance seems largely related to executive functions (Gonneaud et al. 2011; Kliegel et al. 2003; Martin et al. 2003), which depend on the integrity of frontal areas that are also particularly sensitive to the effects of age (Kalpouzos et al. 2009).

The neural substrates of PM were mostly explored in young adults using EBPM paradigms. Studies highlighted the involvement of the rostral prefrontal cortex (RPFC), whose lateral part is activated, contrasting with the deactivation of the medial part (Burgess et al. 2001; 
Burgess et al. 2003; den Ouden et al. 2005; Simons et al. 2006; Rusted et al. 2011; Benoit et al. 2012; Barban et al. 2013; Beck et al. 2014; see Burgess et al. 2011; Cona et al. 2015 for reviews). According to the Gateway Hypothesis (Burgess et al. 2007), the deactivation of the medial part of the RPFC and the activation of its lateral part reflect the shift of attentional focus from external stimulation (i.e. ongoing task) to internal thoughts (i.e. maintenance of the intention). The involvement of other regions, such as the precuneus, the parietal lobe, the anterior and posterior cingulate cortex, the temporal cortex and the insula has been frequently reported but the role of these different regions in PM remains unclear (Burgess et al. 2011). The handful of studies using TBPM paradigms revealed greater involvement of the prefrontal cortex (Momennejad and Haynes 2012; Okuda et al. 2007), consistent with the hypothesis of a higher requirement of self-initiated processes during TBPM (Craik 1986). Oksanen and collaborators (2014) pointed out that prefrontal activity (i.e. reflecting monitoring processes) in TBPM, contrary to EBPM, was not sustained but only transient during clock-checks, probably reflecting the involvement of anticipatory/planning processes. In a previous study in young adults, we directly compared the neural substrates of EBPM and TBPM and showed that they share a common neural network (i.e., activation of the posterior frontal and parietal cortices, deactivation of the medial RPFC) but also have specific features reflecting the different strategies used to perform each task. Thus, we reported higher involvement of posterior cortical areas in EBPM, reflecting visual search of the cue, and higher activity in the dorsolateral prefrontal cortex and the cuneus/precuneus, reflecting time estimation processes in TBPM (Gonneaud et al. 2014).

Only a few studies were conducted in older adults, focusing almost exclusively on EBPM. Studies using event-related potentials (ERP; West 2011 for review), provided mixed results, some of them showing changes in brain activity in older adults (West and Bowry 2005), while others did not (Mattli et al. 2011). Only one ERP study focused on TBPM and 
highlighted changes in older adults over prefrontal areas, as well as a posterior-anterior shift in the distribution of the P3 wave, related to lower accuracy (Cona et al. 2012). This result is in line with previous studies showing increased activity in frontal regions in aging, associated with reduced activity in occipital areas, as described in the Posterior-Anterior Shift in Aging model (PASA, Davis et al. 2008). Other models have been proposed to explain the agerelated functional changes in aging, either in line with a compensatory hypothesis, as the HAROLD model (Hemispheric Asymmetry Reduction in OLDer adults; Cabeza 2002), or not, like in the dedifferentiation hypothesis (Baltes and Lindenberger 1997; Reuter-Lorenz 2002; Reuter-Lorenz and Lustig 2005 for reviews) which rather suggests difficulties for older adults to use specific and selective neural substrates to execute a cognitive task. Another feature of the aging brain that could be critical to PM is the difficulty of older adults to deactivate some areas when performing a cognitive task (Hafkemeijer et al. 2012; Lustig et al. 2003). This may be particularly relevant considering the importance of the deactivation of the medial RPFC in PM. Actually, a difficulty to disengage the medial RPFC in PM would indicate that the decline of PM may be due, at least in part, to a difficulty to shift their focus from external events (i.e. ongoing task) to their internal thoughts (i.e. maintenance of an intention).

Only two studies assessed directly the effects of aging on PM. Gao et al. (2014) found increased brain activity during EBPM, notably in frontal and parietal areas, in the supplementary motor area and fusiform gyrus, as well as in the precuneus. Nonetheless, small samples size, difference in education level between groups, as well as the characteristic of the task (i.e. highly salient cue) make difficult the generalization of this result. Recently, Peira et al. (2016) showed, in older adults, reduced performance in conditions with high demands on prospective and working memory, subserved by an inability to recruit PM-related brain areas. 
In this context, our study aimed unravelling the neural substrates of EBPM and TBPM maintenance in older adults, using fMRI. To do so, we used an fMRI task previously validated in young adults for being able to distinguish between EBPM and TBPM maintenance substrates (i.e. target checking and time estimation respectively). We hypothesized that both the dysfunction of the frontal cortex and a difficulty to deactivate some brain areas (notably the medial RPFC) would subserve the age-related impairment of PM, this one being probably greater for TBPM than for EBPM.

\section{Material and Methods}

\section{Participants}

Twenty healthy young (aged from 18 to 35) and 20 healthy older (aged from 51 to 76) community dwelling adults were enrolled in this study (Table 1). All participants had normal or corrected-to-normal vision, were French-native speakers and right-handed, according to the Edinburgh inventory (Oldfield 1971). All individuals had at least seven years of schooling and the two groups were matched for this parameter. We ensured that they did not have any antecedent of neurological or psychiatric disorders. In addition, older participants were screened for cognitive deficits with the Mini Mental State Examination (MMSE; Folstein et al. 1975) and the Mattis dementia rating scale (Mattis 1976) and only those who had normal scores ( $\geq 27$ and $\geq 136$ respectively) were included. The study was approved by the regional ethics committee (CCP Nord Ouest III) and all participants gave their written informed consent prior to participation.

fMRI tasks 
The PM task has been fully detailed elsewhere (Gonneaud et al., 2014) and was inspired by the design devised by Reynolds et al. (2009), featuring a succession of short blocks with different instructions.

General procedure and ongoing task. Participants had to complete blocks of a semantic categorisation task of pictures (called hereafter the ongoing task) and, for some blocks, a PM instruction was added (see below). Three experimental conditions were implemented: ongoing task only (OG-only), EBPM and TBPM. A fourth condition was used to assess the retrospective component of EBPM, but will not be developed in the present paper. Before the scanning session, subjects were trained to each condition in order to avoid failures due to misunderstanding of the procedure during the fMRI session (Gonneaud et al. 2014; Reynolds et al. 2009; see also Simons et al. 2006). Functional MRI data were acquired in three runs, each of them containing one block of the four conditions (i.e. OG-only, EBPM and TBPM, as well as the retrospective component of EBPM condition). As a result, three blocks of each condition were proposed. The order of presentation of each condition was randomized between runs. After the scanning session, a debriefing was proposed to assess participants' strategies or difficulties during the tasks.

Pictures were displayed within a 280 x 280 pixel white square, bordered by a 20-pixel coloured line. The border colour changed randomly between pictures and could be of nine different colours. In each condition, were also displayed a digital clock in the upper righthand corner (necessary for the TBPM condition) and a forefinger-category association reminder at the bottom of the screen, to avoid any failure due to the difficulty to remember the correct answer keys.

As a whole, subjects were asked to classify 480 colour pictures as "natural" or "manmade" items. Each block was composed of 40 pictures, and subjects had to rate 12 blocks of pictures (including 3 blocks assessing the retrospective component of EBPM, not addressed here). 
Each block was built as follows (Fig. 1): an instruction inviting participants to categorise items as "man-made" or "natural" was first displayed on the screen during 8 seconds. After the instruction, participants were asked to prospectively judge their ability to complete the block on a five-point scale, from very badly to very well. The question remained on the screen during 8 seconds. This metamemory measure was not part of the PM task but was used to introduce a delay between PM instructions and the beginning of the ongoing task, minimizing the possibility for the subject to maintain the instruction in their working memory (McDaniel and Einstein 2007). After the 8 seconds had elapsed, a fixation cross was displayed for 1 second, followed by a block of 40 trials. For each trial, pictures remained on the screen for two seconds, followed by a mask (i.e. random black and white draughtboard delineated by a multi-coloured border) for one or two seconds. Participants were asked to answer as quickly and accurately as possible whether the item displayed was "man-made" or "natural" by pressing with their forefingers. Half of the participants had to press right for man-made items and left for natural ones. Response keys were reversed for the other half of participants. Answers were recorded during the whole trial (picture + mask). The order of presentation of pictures was entirely randomised between individuals. After the 40 trials, they were asked to evaluate retrospectively their performance for this block on the same five-point scale than before (from very badly to very well). After 8 seconds, a fixation cross appeared and a new block began.

PM tasks. Both groups were instructed that we were also interested in their ability to remember doing something in the future (Fig. 1). Consequently, for some blocks, instructions required to give an additional answer, using the middle-finger (the right one for half of the participants, the left for the other half), in response to a specific item/time interval. In the EBPM condition, participants were asked to press with the additional button when they saw a specific colour of border (e.g. press for blue border). In the TBPM condition they had to press 
with their middle-finger every 30 seconds. To do the TBPM task, the participant had at their disposal the digital clock, displayed in the upper right corner of the screen in each condition. Such a choice allowed us to ensure that comparison between TBPM and EBPM was not biased by activity due to the press of an additional button to check a hidden clock, the maintenance of an additional key-answer association, or to the additional visual stimulation in TBPM. This was particularly relevant regarding older adults' difficulties in executive functioning, notably in their abilities to manage multiple tasks. To minimize the impact of the presence of the clock, it was displayed in the upper right-hand corner of the screen so that subjects needed to voluntarily direct their look to monitor time. Debriefing questionnaires (not shown) allowed us also to ensure that subjects did not look at the clock during the whole experiment as they reported, especially young participants, having monitored the clock in a classic "J-shaped" frequency (a little at the beginning and more often at the end). A total of five PM answers were expected by block. For PM trials, both the PM and the ongoing task answers were required. No order of answer was imposed to the participants, first to avoid, with such a stringent instruction, any additional cost that we noticed in the pretesting phase of this work, especially for older subjects (see also Ihle et al. 2013). To better control for visual stimulation between blocks, the clock was displayed in the three conditions (even in OG-only and EBPM) and the colour border of pictures changed between trials, even in OG-only and TBPM conditions. To mimic the fact that the picture to rate and the cue in the EBPM condition always appeared jointly, the end of the 30 seconds intervals in TBPM always coincided with the appearance of a new trial. Conversely, to mimic frequency of TBPM, in the EBPM condition the cue appeared once per 30 seconds interval, but not every 30 seconds to avoid strategies based on predicting the time of appearance of the cues. We acknowledge that the presence of the digital clock on the screen during the entire experience is not the optimal way to assess TBPM and the presence of the clock, even in the peripheral 
environment, may be considered by some as an EBPM task rather than a TBPM one. Consequently, the following results should be taken with caution regarding this alternative view and will be discussed in this sense later.

\section{fMRI data acquisition}

Two scanning sessions were performed on a 3T Philips Achieva scanner (Eindhoven, The Netherlands). During the first session, a T1-weighted MRI scan was acquired (3D-T1-FFE sagittal, $\mathrm{TR}=20 \mathrm{~ms} ; \mathrm{TE}=4.6 \mathrm{~ms} ;$ flip angle $=10^{\circ} ; 180$ slices; slice thickness $=1 \mathrm{~mm}$; matrix $=256 \times 256$; acquisition voxel size $=1 \times 1 \times 1 \mathrm{~mm}^{3}$ ), followed by a high resolution $\mathrm{T} 2-$ weighted anatomical image (2D-T2-SE sagittal, $\mathrm{SENSE}$ factor $=2 ; \mathrm{TR}=5500 \mathrm{~ms}$; TE $=80$ $\mathrm{ms}$; flip angle $=90^{\circ} ; 81$ slices; slice thickness $=2 \mathrm{~mm} ;$ matrix $=256 \times 256$; acquisition voxel size $=2 \times 1 \times 1 \mathrm{~mm}^{3}$ ) and a non-EPI (Echo Plannar Imaging) T2-Star image (2D-T2 Star-FFE axial, $\mathrm{SENSE}$ factor $=2 ; \mathrm{TR}=3509 \mathrm{~ms} ; \mathrm{TE}=30 \mathrm{~ms}$; flip angle $=90^{\circ} ; 70$ slices; slice thickness $=2 \mathrm{~mm}$; matrix $=128 \times 128$; acquisition voxel size $\left.=2 \times 2 \times 2 \mathrm{~mm}^{3}\right)$. In the second acquisition, a non-EPI T2-Star image, similar to the anatomical session's one, was acquired. Functional data were then acquired using an interleaved 2D T2-Star EPI sequence designed to reduce geometrical distortions and magnetic susceptibility artefacts (2D-T2 Star-FFE-EPI axial, $\operatorname{SENSE}$ factor $=2 ; \mathrm{TR}=2600 \mathrm{~ms} ; \mathrm{TE}=30 \mathrm{~ms} ;$ flip angle $=80^{\circ} ; 46$ slices; slice thickness $=3.0 \mathrm{~mm} ;$ matrix $=80 \times 80 ;$ acquisition voxel size $=2.8 \times 2.8 \times 3.0 \mathrm{~mm}^{3} ; 266$ volumes per run). Three runs of functional imaging were acquired during the session. The first six volumes of each run were discarded to control for magnetic saturation effects.

Behavioural data analysis

To assess the effects of aging on the different experimental conditions, analyses of variance (ANOVAs) were conducted on accuracy and reaction times. First, to determine whether 
young and older adults were as accurate in answering to PM items for EBPM and TBPM, a 2 x 2 ANOVA was conducted on correct responses to PM items (i.e. presses of middle-finger) with group (young vs older) as between-subjects factor and nature of the PM task (EBPM vs TBPM) as within-subjects factor. For TBPM, answers were considered as correct if they were made during the trial corresponding to the target time (i.e. during the picture or the mask). Second, we assessed PM interference effect on the ongoing task by comparing accuracy and reaction times for the semantic categorisation task between the conditions without PM (OGonly) and those in which an intention was added (EBPM and TBPM). To do so, $2 \times 3$ ANOVAs with group (young vs old) as between-subjects factor and condition (OG-only vs EBPM vs TBPM) as within-subjects factor were conducted both on the percent of correct answers to ongoing items and reaction times (only for correct answers) in each condition. Tukey's HSD post hoc comparisons were performed when applicable.

fMRI data processing and analysis

fMRI data were analysed using SPM 5 () implemented in Matlab7.4 (Mathworks Inc., MA). Preprocessing was conducted as follows: EPI data were checked to ensure the absence of artifacts and realigned with the first volume of the first run. Then, to control for geometric EPI distortions, the mean EPI image was coregistered onto the non-EPI T2-Star volume of the functional session, the non-EPI T2-Star volume of the functional session was coregistered onto the anatomical one, the non-EPI T2-Star volume of the anatomical session was then coregistered onto the T2 image, and finally the T2 volume was coregistered onto the T1 image according to the procedure described by Villain et al. (2010). T1 was segmented/normalized on the Montreal Neurological Institute (MNI) template and resulting parameters were applied to the coregistred T1, non-EPI T2-star volumes and EPI images. Finally, EPI images were smoothed using an 8-mm FWHM Gaussian kernel. 
A General Linear Model was used to assess the effects of group (young vs older) and experimental condition (OG-only vs EBPM vs TBPM). In a first level (individual analysis), each trial (picture + mask) was specified individually; instructions, questions and errors were modeled as regressors of no interest. An event-related design has been used so that, for each participant, main effects were estimated for OG-only, EBPM and TBPM conditions, specifying the onset and the full duration of every trials of the conditions (ongoing items and PM items) conducing to a correct answer. Correct responses on OG items and on PM items were modeled separately at the first level. For PM items, both correct ongoing answers and correct PM answers were required. Finally, to account for potential differences in response times between conditions, response times for each item were added in the model as a parametric modulator.

In a second, group-level analysis, OG-only, EBPM and TBPM for both groups were entered in the same model, allowing comparisons between each condition, group and their interaction. First, we wanted to highlight, in the older group, the neural substrates of each PM condition, independently from each other. To do so, subtractions between each PM condition and the OG-only condition were performed. Second, to assess age-related changes in PM activity, interactions between group (young vs old) and condition (OG-only vs PM) were assessed for EBPM and TBPM separately. In addition, the interaction between group (young vs old) and the nature of the PM task (EBPM vs TBPM) was assessed to evaluate age-related changes in EBPM and TBPM specificities. When an interaction was found, post-hoc analyses were conducted extracting BOLD signal values from each region and using Tukey's HSD tests. Finally, to determine whether the relationship between brain activity and performance was comparable or not between the young and older participants, interactions between Group and BOLD signal values were assessed. Then, correlations between BOLD signal values and 
accuracy on PM items were conducted, focusing on the older group, to determine whether brain activity observed can predict their PM performance.

Statistical maps were thresholded at $p<0.001$ uncorrected at the voxel level and a minimal $\begin{array}{lllll}\text { cluster size } & \text { was } & \text { calculated } & \text { using } & \text { 3dClustSim }\end{array}$ (http://afni.nimh.nih.gov/pub/dist/doc/program_help/3dClustSim.html) to obtain a significance level corrected for multiple comparisons. Thus, the probability of false positive for the entire functional volume was $p<0.05$, as estimated by Monte Carlo simulations, resulting in a minimum cluster-size of 69 voxels.

\section{Results}

Behavioral results

Accuracy and response times are reported in Table 2. While reaction times were normally distributed, accuracy scores were not. Consequently, nonparametric statistics were used to assess the effects of group and condition on accuracy scores, using Kolmogorov-Smirnov and Wilcoxon tests for between-group and within-group comparisons, respectively. As results from nonparametric analyses (data not shown) and parametric analyses (ANOVAs followed by post-hoc analyses when applicable) were similar, we decided to present results from parametric analyses only, both for simplifying and homogenizing the presentation of results. Concerning PM tasks, the comparison of correct presses of middle-finger in each condition revealed a main effect of group, with younger adults more accurate than the older ones $\left(F_{(1,38)}\right.$ $=27.56 ; p<0.001 ; \eta^{2}$ partial $\left.=0.42\right)$, no main effect of condition $\left(F_{(1,38)}=2.58 ; p=0.12 ; \eta^{2}\right.$ partial $=0.06)$ and a marginal interaction between the two factors $\left(F_{(1,38)}=3.87 ; p=0.06 ; \eta^{2}\right.$ partial $=$ 0.09). As the interaction was close to the significance threshold and given our a priori hypothesis of a greater effect of age on TBPM compared to EBPM, Tukey's HSD post hoc comparisons were conducted. These analyses showed that older participants were 
significantly less accurate than younger individuals for TBPM $(p<0.001)$ but not for EBPM $(p=0.13)$. The effect of condition was not significant in younger adults $(p=0.99)$, while older participants tended to perform better for EBPM than TBPM $(p=0.07)$.

To assess the interference of PM on the ongoing task, ANOVAs were also conducted on correct answers for ongoing items in each condition (OG-only vs EBPM vs TBPM). This analysis revealed a main effect of group $\left(F_{(1,38)}=15.20 ; p<0.001 ; \eta^{2}\right.$ partial $\left.=0.29\right)$, a main effect of condition $\left(F_{(2,76)}=9.40 ; p<0.001 ; \eta^{2}\right.$ partial $\left.=0.20\right)$ and a significant interaction between both factors $\left(F_{(2,76)}=5.7 ; p<0.005 ; \eta^{2}\right.$ partial $\left.=0.13\right)$. Tukey's HSD post hoc comparisons revealed that the younger group outperformed the older one on the ongoing items for EBPM and TBPM conditions ( $p s<0.01$ ), but not in the OG-only condition ( $p=$ 0.99). While the younger group had similar performance in each condition ( $p s=0.99)$, older adults were less accurate on the categorisation task in EBPM and TBPM compared to the OG-only condition ( $p s<0.001)$, with no difference between EBPM and TBPM $(p=0.99)$.

A similar analysis on response times also revealed a main effect of group $\left(F_{(1,38)}=20.45 ; p<\right.$ $0.001 ; \eta^{2}$ partial $\left.=0.35\right)$, a main effect of condition $\left(F_{(2,76)}=80.27 ; p<0.001 ; \eta_{\text {partial }}^{2}=0.68\right)$ and a significant interaction between these factors $\left(F_{(2,76)}=8.42 ; p<0.001 ; \eta^{2}\right.$ partial $\left.=0.18\right)$. Tukey's HSD post hoc comparisons showed that the older group tended to be slower in the OG-only condition $(p=0.07)$, and was also slower in TBPM and EBPM $(p s<0.001)$. Finally, younger adults were significantly slower in the EBPM condition than in the two other conditions (OG-only and TBPM; ps < 0.001), without any speed difference between these two latter $(p=0.37)$. For older adults, response times were slower in EBPM than TBPM ( $\mathrm{p}<0.05)$, and slower in both PM conditions compared to the OG-only one ( $p s<$ 0.001). These results indicate that maintaining an intention has a cost on the execution of the ongoing task, especially in older adults. 
fMRI data

Neural substrates of EBPM and TBPM in young adults

Patterns of brain activity for EBPM and TBPM in young healthy adults are fully described in a previous paper (Gonneaud et al. 2014), and the neural substrates highlighted in the present study (using a different statistical model, including notably a second group of participants) were very similar to those reported in the initial study. Briefly, adding an intention to the ongoing task led in both conditions (i.e. conjunction analyses; data not shown) to increased activity in frontal, parietal and occipital regions as well as in the insula, together with a deactivation of medial areas, notably the medial RPFC, the precuneus, the left angular gyrus and the right middle temporal pole. In addition, direct comparison of EBPM and TBPM conditions in young participants revealed that left posterior cortical areas were more involved in EBPM, while the right dorsolateral gyrus, cuneus and the left cerebellum regions were more activated in TBPM.

\section{Neural substrates of EBPM and TBPM in healthy older adults}

PM conditions were first compared to OG-only (contrast 'OG-only minus PM condition') to highlight brain areas deactivated during PM. Interestingly, in older adults, no region was deactivated in PM relative to OG-only, neither for EBPM nor for TBPM.

The reverse contrasts ('PM conditions minus OG-only') revealed that adding an intention led to increased activity in a large set of brain areas. A bilateral set of regions including the cuneus, extending to the lingual and middle occipital gyri, the middle frontal cortex extending to parietal regions, the insula and the thalamus, was significantly more activated during EBPM than OG-only (Fig.2, left panel).

As for TBPM, higher activity relative to the OG-only condition was found, bilaterally, in the lingual gyrus extending to the middle occipital gyrus, the superior and inferior parietal 
lobules, and the insula. Frontal activations were also found in the middle and superior gyri (Fig.2, middle panel).

Finally, no difference was found between EBPM and TBPM conditions (Fig.2, right panel).

\section{Interaction between age and PM}

First, an analysis assessing the differential effect of age on the EBPM vs OG-only conditions revealed an interaction in the left middle and right inferior frontal, left superior occipital, left superior temporal, bilateral supplementary motor area, precentral regions, right paracentral lobule and the precuneus. Extracting BOLD signal values from each cluster highlighted that, compared to young participants, older adults showed much more activation in some clusters and much less deactivation in the others (Fig.3). More specifically, older adults exhibited a significant increase in activity during EBPM compared to the OG-only condition in the bilateral supplementary motor area, left inferior and right middle frontal, superior parietal and occipital cortices. These differences were not observed in young participants, suggesting that the older group recruit additional brain regions, which are not specific to the task. The left precentral region showed also a significant increase during EBPM compared to OG-only in the older group, but the increased activation was also significant in the younger group, suggesting in this case a higher recruitment of relevant regions in older participants. In a second group of regions, including the precuneus, the left middle temporal and right paracentral lobule, a significant decrease of activity in EBPM compared to OG-only was found in the younger group (i.e. deactivations), but not in the older group.

A similar analysis assessing the differential effect of age on TBPM vs OG-only conditions revealed an interaction in the right precuneus and bilateral supplementary motor area. Patterns of brain activity were similar to those highlighted in EBPM (Fig.3) with 
regions showing in the older group more activity in the TBPM than in the ongoing condition (supplementary motor area) and regions with no significant deactivation during TBPM (right precuneus) compared to young participants.

Finally, no interaction was found when assessing the differential effect of age on the EBPM vs TBPM conditions.

\section{Correlation between PM performance and brain activity in older vs younger adults}

The results reported above suggest that, in older adults, maintenance of an intention is mainly associated with the activation of additional brain areas and difficulties to deactivate other regions. To better understand the relationship between functional activity and PM performance, accuracy scores were correlated with mean BOLD signal values extracted from the clusters we found to be more activated in the older group, those that older adults failed to deactivate, and regions commonly activated by young and older adults. No interaction was found on PM accuracy between Group (young vs older) and BOLD signal values, regardless of the region assessed. Finally, no significant correlation was found, in older adults, between EBPM or TBPM accuracy and the level of activity in the regions previously described.

\section{Discussion}

Using fMRI, we investigated in older adults the neural substrates of both event-based (EBPM) and time-based (TBPM) prospective memory. Our data mainly revealed an agerelated impairment of PM, more specifically for TBPM, and the recruitment of additional regions in older adults for PM maintenance together with difficulties to deactivate some brain areas. 
Behavioural data revealed a decline of PM in older adults compared to young ones, more pronounced for TBPM. The greater sensitivity of TBPM to the effects of age, in line with most of the previous reports (Einstein et al. 1995; Park et al. 1997), is particularly interesting because, in the present study, the two conditions have been made as similar as possible, differing only on the kind of strategic monitoring of the environment that should be engaged (check for the appearance of a non-focal cue or for the appropriate time to answer, in EBPM and TBPM conditions respectively). This suggests that, in these conditions, the TBPM task required older participants to engage more self-initiated processes (Craik 1986). More specifically, TBPM may engage more self-initiated processes than EBPM, despite the fact that, in conditions where TBPM and EBPM tasks are not made as similar as possible, the reverse pattern could be observed (see for example Gonneaud et al., 2011). Furthermore, older individuals demonstrated a higher cost of adding an intention on both accuracy and response times for the ongoing task (i.e., "natural"/"man-made" judgment) compared to young adults. In this latter group, a slowdown was only observed in EBPM. Because in TBPM the moment of retrieval is predictable, strategic monitoring was judged to be only transient and less costly (Gonneaud et al. 2014). For the older group, this cost of PM was observed on both accuracy and response times, and almost equivalent in EBPM and TBPM. It suggests that strategic monitoring is particularly costly for older participants who also, unlike young participants, did not benefit from the predictability of the TBPM intention and had greater difficulties to monitor time. This seems to be in line with reports obtained during the debriefing session proposed just after the fMRI task. Indeed, young participants reported both automatic retrieval and constant monitoring in the same proportion (50/50) for EBPM while the majority of them reported a different strategy in TBPM. Indeed, 70\% reported a periodic checking of time, including the classical "J-shaped" curve strategy (see for example Khan et al. 2008; Occhionero et al. 2010), and only 30\% reported sustained or constant clock 
monitoring. By contrast, the proportion reporting having relied on a constant checking of the clock in TBPM is higher in older participants (50\%; data not shown). This non-optimal monitoring, mainly in TBPM, may contribute to reduced PM performance (Oksanen et al. 2014). However, as the responses profiles did not statistically differ between groups, these elements need to be taken cautiously

The analysis of fMRI data revealed different neural substrates for the maintenance of intentions between older and young individuals, as previously reported using ERP (Cona et al. 2012; West and Bowry 2005). In young individuals, we previously identified a common cerebral network for EBPM and TBPM maintenance involving the activation of frontal and parietal areas, associated with decreased activity in the medial RPFC (Gonneaud et al. 2014), that may reflect the involvement of a retrieval mode (i.e. cognitive state allowing to process stimuli as cue for the retrieval of an intention). In the present study, higher activity was found in older adults during both EBPM and TBPM in several brain areas (notably in frontal and parietal areas as well as in the supplementary motor area and the precuneus), while no deactivation was found. Looking in more details at the effect of age on PM-related activity by means of interaction analyses, it appeared that during PM tasks (compared to the OG-only condition) older adults recruit additional regions, not activated by younger participants and therefore not specific to the PM tasks. These regions include the bilateral supplementary motor area, left inferior and right middle frontal, left superior temporal, superior parietal and occipital cortices as well as the precuneus, that were already found in another study (Gao et al. 2014). It is unlikely that the recruitment of these regions have a compensatory role as no correlation was found between their activity and PM performance. Our results are rather in line with the hypothesis of dedifferentiation in aging (Baltes and Lindenberger 1997; ReuterLorenz and Lustig 2005) and suggest difficulties in older adults to recruit neural networks specific and selective for strategic monitoring in PM. Such difficulties to recruit PM-related 
brain networks were already reported in older adults, in conditions with high demands on prospective and working memory (Peira et al., 2016). However, the "non-compensatory" role of these activations remains to be further explored, at least for EBPM, as no significant difference was found between young and older participants in accuracy, and ceiling effects may represent a limitation when searching for correlation between PM and brain activity. By contrast, no brain area was less activated in older adults compared to young individuals, suggesting that older adults correctly recruited the critical regions to perform the PM task, but were not able to select only these regions. The absence of differential relationship between activity and performance in young $v s$ older adults prevents going further in the interpretation of the different mechanisms underlying PM performance in older adults. However, this lack of interaction may be attributed to the weak statistical power due to ceiling effects and a limited variability of performance.

Considering the critical involvement of medial RPFC deactivation in PM maintenance (Burgess et al. 2001; Simons et al. 2006), we also focused our interest on PM-related deactivations. According to the Gateway Hypothesis (Burgess et al. 2007), medial RPFC deactivation would reflect the disengagement from stimulus-oriented attending to intention maintenance. Interestingly, our analyses showed no deactivation of the medial RPFC in older adults during EBPM and TBPM tasks, while it was observed in young participants in both conditions. This could reflect a difficulty for older adults to disengage their attention from external stimulations to focus on intention maintenance. This may have increased their difficulty to remember and correctly execute PM intentions. Interaction analyses revealed that older adults were impaired in their ability to deactivate the medial RPFC, but also various regions including the precuneus, the left middle temporal and right paracentral lobule, during PM conditions. This difficulty to deactivate some brain regions in older individuals has already been reported in several studies using notably verb generation or n- 
back tasks (Persson et al. 2007; Prakash et al. 2012). When engaged in a cognitive task, young subjects classically demonstrate deactivation of the Default Mode Network, including the dorsal and ventral medial prefrontal cortices, the posterior cingulate gyrus and precuneus, the lateral parietal cortices and medial temporal lobes. Reduced deactivations observed here in older participants can be interpreted as a difficulty for those subjects to switch from this default mode to a task-related mode (Mevel et al. 2011), which may impact noticeably on strategic monitoring processes. Once again, this difficulty to disengage inappropriate regions to the task is in favour of the dedifferentiation hypothesis.

We previously reported specific patterns of activation for EBPM and TBPM in young adults. Maintenance of EB intentions elicited activity in occipital areas, reflecting target checking, while TBPM activated a right-sided network, notably the dorsolateral prefrontal cortex and the cuneus/precuneus, reflecting the involvement of time estimation processes (Gonneaud et al. 2014). The same comparisons in older participants did not reveal such functional specialization according to the nature of the task. However, no direct interaction was found between group and nature of the PM condition, except at a very permissive threshold (data not shown). Even if it remains to be confirmed, this result, together with the analysis restricted to the older group, lends support to the dedifferentiation hypothesis of the cerebral networks in aging. These difficulties to use efficient strategic monitoring processes and dedicated neural networks according to the requirements of PM tasks may have contributed to decreased PM performance.

Considering our behavioural and fMRI data in the context of the Compensation-Related Utilisation of Neural Circuit Hypothesis (CRUNCH; Reuter-Lorenz and Cappell 2008; Reuter-Lorenz and Park 2010) could open some perspectives to this work. In fact, this model posits that for low-demanding tasks, older adults may increase activity in some brain areas to compensate, successfully, their difficulties. In contrast, for more complex tasks, they could 
not fulfill tasks demands any longer, and their functional activity cannot be compensatory anymore. In this context, our data suggest that the age-related decline in PM may be related to the high demand of the tasks, for which older adults cannot fully compensate by increased activations. As a result, they engage nonspecific regions to execute the task, as revealed by the activation of brain areas that were not engaged by young participants and a difficulty to deactivate other regions. This seems particularly true for TBPM. We suggest that the higher age-related decline on TBPM compared to EBPM is related to the fact that the former is more demanding for older adults, who consequently have higher difficulty to use the appropriate cognitive and neural networks in order to compensate. However, the lack of direct evidence for compensation in EBPM (i.e. no evidence of performance/brain activity correlation, maybe related to ceiling effects) limits this interpretation. Future studies are needed contrasting lowand highly-demanding tasks, to better underlie compensation networks in older adults.

We must acknowledge that, despite our previous study revealing partially different neural substrates for EBPM and TBPM, assessed using the same tasks (Gonneaud et al., 2014), further studies are needed to assess more properly TBPM. Indeed, we did not use a hidden clock that would appear only when subjects press on a specific key. This choice was notably driven by the fact that adding another response button to display a clock would have increased the complexity of the task for older participants. However, considering the presence of the clock during the entire experiment, our TBPM task may be alternatively viewed as assessing EBPM. In this context, our results may be interpreted as reflecting the mechanisms related to two different EBPM tasks. Thus, our results would not be specific to the distinction between EBPM and TBPM but would rather argue on partially different processes that would underlie an EBPM task mainly relying on self-initiated processes (“clock-based EBPM") and a less costly EBPM task (“colour-based" one). Future studies should definitively assess more deeply, and with more specific designs, the effect of aging on 
TBPM cerebral substrates and decide between the two different points of view. The use of an hidden clock should particularly increase the ecological value of the paradigm and provide a measure of monitoring in TBPM. Additionally, the delay introduced between each PM instruction and the realization of the task (i.e. 8 seconds) is quite short and longer delays are usually recommended (McDaniel \& Einstein, 2007). This is to note that similar design, in which there was even no delay between instructions and PM assessment, had been conclusively used in previous studies (Reynolds et al., 2009, Peira et al., 2016). As a result, we are quite confident that such a short delay does not have a major effect on our results. However, future studies may focus on PM tasks that better suits the recommendations regarding this point.

To sum-up, this study was the first to assess directly the neural substrates of EBPM and TBPM in healthy aging using fMRI. In line with current models of cerebral and cognitive aging (dedifferentiation hypothesis and $\mathrm{CRUNCH}$ model), our results suggest that older individuals have troubles in selecting appropriate strategies to perform PM tasks, subserved by difficulties to recruit relevant neural networks. These difficulties manifest as noncompensatory recruitment of additional regions coupled with a difficulty to deactivate other brain regions to execute PM tasks and an inability to select specific brain areas for EBPM and TBPM. Further studies are needed to achieve a better understanding of the cerebral mechanisms explaining the decline of PM in healthy aging, in particular for TBPM decline for which the experimental design could be improved to assess more finely the richness of this concept. More specifically, the use of a PM task with less cognitive demands (e.g. focal task) may be of particular interest, in the framework of the CRUNCH model, to reveal the patterns of brain activity that allow older adults to effectively compensate for their PM difficulties. 


\section{Compliance with Ethical Standards}

Conflict of interest: The authors declare that they have no conflict of interest.

Ethical approval: All procedures performed in studies involving human participants were in accordance with the ethical standards of the institutional and/or national research committee and with the 1964 Helsinki declaration and its later amendments or comparable ethical standards.

Informed consent: Informed consent was obtained from all individual participants included in the study.

Acknowledgments: The authors would like to thank F. Lamberton for his help in the fMRI sequences, J. Chavant, J. Dayan, C. Lebouleux, M.H. Noel, M.C. Onfroy, A. Quillard and C. Schupp for data acquisition and recruitment of participants.

Fundings: This work was supported by the Association France-Alzheimer and the French Ministère de l'Enseignement Supérieur et de la Recherche. 


\section{References}

Baltes, P. B., \& Lindenberger, U. (1997). Emergence of a powerful connection between sensory and cognitive functions across the adult life span: A new window to the study of cognitive aging? Psychology and Aging, 12(1), 12-21.

Barban, F., Carlesimo, G. A., Macaluso, E., Caltagirone, C., \& Costa, A. (2013). Functional brain activity within the medial and lateral portion of BA10 during a prospective memory task. Behavioural Neurology, 26(3), 207-209.

Beck, S. M., Ruge, H., Walser, M., \& Goschke, T. (2014). The functional neuroanatomy of spontaneous retrieval and strategic monitoring of delayed intentions. Neuropsychologia, 52, 37-50.

Benoit, R. G., Gilbert, S. J., Frith, C. D., \& Burgess, P. W. (2012). Rostral prefrontal cortex and the focus of attention in prospective memory. Cerebral cortex (New York, N.Y.: 1991), 22(8), 1876-1886.

Burgess, P. W., Dumontheil, I., \& Gilbert, S. J. (2007). The gateway hypothesis of rostral prefrontal cortex (area 10) function. Trends in cognitive sciences, 11(7), 290-298.

Burgess, P. W., Gonen-Yaacovi, G., \& Volle, E. (2011). Functional neuroimaging studies of prospective memory: what have we learnt so far? Neuropsychologia, 49(8), 2246-2257.

Burgess, P. W., Quayle, A., \& Frith, C. D. (2001). Brain regions involved in prospective memory as determined by positron emission tomography. Neuropsychologia, 39(6), $545-555$.

Burgess, P. W., Scott, S. K., \& Frith, C. D. (2003). The role of the rostral frontal cortex (area 10) in prospective memory: a lateral versus medial dissociation. Neuropsychologia, 41(8), 906-918.

Cona, G., Arcara, G., Tarantino, V., \& Silvia Bisiacchi, P. (2012). Age-related differences in the neural correlates of remembering time-based intentions. Neuropsychologia, 50(11), 
2962-2704.

Cona, G., Scarpazza, C., Sartori, G., Moscovitch, M., \& Bisiacchi, P. S. (2015). Neural bases of prospective memory: a meta-analysis and the "Attention to Delayed Intention" (AtoDI) model. Neuroscience and Biobehavioral Reviews, 52, 21-37.

Craik, F. I. M. (1986). A functional account of age differences in memory. In F. Lix \& H. Hagendorf (Eds.), Human memory and cognitive capabilities: Mechanisms and performances (pp. 409-422). Amsterdam: Elsevier Science.

Den Ouden, H. E. M., Frith, U., Frith, C., \& Blakemore, S.-J. (2005). Thinking about intentions. NeuroImage, 28(4), 787-796.

Einstein, G. O., \& McDaniel, M. A. (1990). Normal aging and prospective memory. Journal of Experimental Psychology. Learning, Memory, and Cognition, 16(4), 717-726.

Einstein, G. O., McDaniel, M. A., Richardson, S. L., Guynn, M. J., \& Cunfer, A. R. (1995). Aging and prospective memory: examining the influences of self-initiated retrieval processes. Journal of Experimental Psychology. Learning, Memory, and Cognition, 21(4), 996-1007.

Folstein, M. F., Folstein, S. E., \& McHugh, P. R. (1975). "Mini-mental state". A practical method for grading the cognitive state of patients for the clinician. Journal of psychiatric research, 12(3), 189-198.

Gao, J., Cheung, R. T. F., Chan, Y.-S., Chu, L.-W., Mak, H. K. F., \& Lee, T. M. C. (2014). The relevance of short-range fibers to cognitive efficiency and brain activation in aging and dementia. PloS One, 9(4), e90307.

Gonneaud, J., Kalpouzos, G., Bon, L., Viader, F., Eustache, F., \& Desgranges, B. (2011). Distinct and shared cognitive functions mediate event- and time-based prospective memory impairment in normal ageing. Memory (Hove, England), 19(4), 360-377.

Gonneaud, J., Rauchs, G., Groussard, M., Landeau, B., Mézenge, F., de La Sayette, V., et al. 
(2014). How do we process event-based and time-based intentions in the brain? an fMRI study of prospective memory in healthy individuals. Human Brain Mapping, 35(7), 3066-3082.

Guynn, M. J., McDaniel, M. A., \& Einstein, G. O. (1998). Prospective memory: when reminders fail. Memory \& Cognition, 26(2), 287-298.

Hafkemeijer, A., van der Grond, J., \& Rombouts, S. A. R. B. (2012). Imaging the default mode network in aging and dementia. Biochimica et biophysica acta, 1822(3), 431441.

Henry, J. D., MacLeod, M. S., Phillips, L. H., \& Crawford, J. R. (2004). A meta-analytic review of prospective memory and aging. Psychology and Aging, 19(1), 27-39.

Ihle, A., Hering, A., Mahy, C. E. V., Bisiacchi, P. S., \& Kliegel, M. (2013). Adult age differences, response management, and cue focality in event-based prospective memory: a meta-analysis on the role of task order specificity. Psychology and Aging, 28(3), 714-720.

Kalpouzos, G., Chételat, G., Baron, J.-C., Landeau, B., Mevel, K., Godeau, C., et al. (2009). Voxel-based mapping of brain gray matter volume and glucose metabolism profiles in normal aging. Neurobiology of Aging, 30(1), 112-124.

Khan, A., Sharma, N. K., \& Dixit, S. (2008). Cognitive load and task condition in event- and time-based prospective memory: an experimental investigation. The Journal of Psychology, 142(5), 517-531.

Kliegel, M., Ramuschkat, G., \& Martin, M. (2003). [Executive functions and prospective memory performance in old age: an analysis of event-based and time-based prospective memory]. Zeitschrift Für Gerontologie Und Geriatrie: Organ Der Deutschen Gesellschaft Für Gerontologie Und Geriatrie, 36(1), 35-41.

Lustig, C., Snyder, A. Z., Bhakta, M., O’Brien, K. C., McAvoy, M., Raichle, M. E., et al. 
(2003). Functional deactivations: change with age and dementia of the Alzheimer type. Proceedings of the National Academy of Sciences of the United States of America, 100(24), 14504-14509.

Mäntylä, T., Missier, F. D., \& Nilsson, L.-G. (2009). Age differences in multiple outcome measures of time-based prospective memory. Neuropsychology, Development, and Cognition. Section B, Aging, Neuropsychology and Cognition, 16(6), 708-720.

Martin, M., Kliegel, M., \& McDaniel, M. A. (2003). The involvement of executive functions in prospective memory performance of adults. International Journal of Psychology, 38(4), 195-206.

Mattis, S. (1976). Mental status examination for organic mental syndrome in the elderly patient. In L. Bellack \& T. E. Karasu (Eds.), Geriatric psychiatry (pp. 77-121). New York: Grune \& Stratton.

Mattli, F., Zöllig, J., \& West, R. (2011). Age-related differences in the temporal dynamics of prospective memory retrieval: a lifespan approach. Neuropsychologia, 49(12), 34943504 .

McDaniel, M. A., \& Einstein, G. O. (2000). Strategic and automatic processes in prospective memory retrieval: a multiprocess framework. Applied Cognitive Psychology, 14(7), S127-S144.

McDaniel, M. A., \& Einstein, G. O. (2007). Prospective memory: an overview and synthesis of an emerging field. Thousand Oaks (CA): Sage Publications.

McDaniel, M. A., \& Einstein, G. O. (2011). The neuropsychology of prospective memory in normal aging: a componential approach. Neuropsychologia, 49(8), 2147-2155.

Mevel, K., Chételat, G., Eustache, F., \& Desgranges, B. (2011). The default mode network in healthy aging and Alzheimer's disease. International journal of Alzheimer's disease, 2011, 535816. 
Momennejad, I., \& Haynes, J.-D. (2012). Human anterior prefrontal cortex encodes the "what" and "when" of future intentions. NeuroImage, 61(1), 139-148.

Occhionero, M., Esposito, M. J., Cicogna, P. C., \& Nigro, G. (2010). The effects of ongoing activity on time estimation in prospective remembering. Applied Cognitive Psychology, 24(6), 774-791.

Oksanen, K. M., Waldum, E. R., McDaniel, M. A., \& Braver, T. S. (2014). Neural mechanisms of time-based prospective memory: evidence for transient monitoring. PloS One, 9(3), e92123.

Okuda, J., Fujii, T., Ohtake, H., Tsukiura, T., Yamadori, A., Frith, C. D., \& Burgess, P. W. (2007). Differential involvement of regions of rostral prefrontal cortex (Brodmann area 10) in time- and event-based prospective memory. International Journal of Psychophysiology: Official Journal of the International Organization of Psychophysiology, 64(3), 233-246.

Oldfield, R. C. (1971). The assessment and analysis of handedness: The Edinburgh inventory. Neuropsychologia, 9(1), 97-113.

Park, D. C., Hertzog, C., Kidder, D. P., Morrell, R. W., \& Mayhorn, C. B. (1997). Effect of age on event-based and time-based prospective memory. Psychology and Aging, 12(2), 314-327.

Peira, N., Ziaei, M., \& Persson, J. (2016). Age differences in brain systems supporting transient and sustained processes involved in prospective memory and working memory. NeuroImage, 125, 745-755.

Persson, J., Lustig, C., Nelson, J. K., \& Reuter-Lorenz, P. A. (2007). Age differences in deactivation: a link to cognitive control? Journal of cognitive neuroscience, 19(6), $1021-1032$.

Prakash, R. S., Heo, S., Voss, M. W., Patterson, B., \& Kramer, A. F. (2012). Age-related 
differences in cortical recruitment and suppression: implications for cognitive performance. Behavioural brain research, 230(1), 192-200.

Reuter-Lorenz, P. A., \& Cappell, K. A. (2008). Neurocognitive Aging and the Compensation Hypothesis. Current Directions in Psychological Science, 17(3), 177-182.

Reuter-Lorenz, P. A., \& Lustig, C. (2005). Brain aging: reorganizing discoveries about the aging mind. Current Opinion in Neurobiology, 15(2), 245-251.

Reuter-Lorenz, P. A., \& Park, D. C. (2010). Human neuroscience and the aging mind: a new look at old problems. The Journals of Gerontology. Series B, Psychological Sciences and Social Sciences, 65(4), 405-415.

Reynolds, J. R., West, R., \& Braver, T. (2009). Distinct neural circuits support transient and sustained processes in prospective memory and working memory. Cerebral Cortex (New York, N.Y.: 1991), 19(5), 1208-1221.

Rusted, J., Ruest, T., \& Gray, M. A. (2011). Acute effects of nicotine administration during prospective memory, an event related fMRI study. Neuropsychologia, 49(9), 23622368.

Simons, J. S., Schölvinck, M. L., Gilbert, S. J., Frith, C. D., \& Burgess, P. W. (2006). Differential components of prospective memory? Evidence from fMRI. Neuropsychologia, 44(8), 1388-1397.

Villain, N., Landeau, B., Groussard, M., Mevel, K., Fouquet, M., Dayan, J., et al. (2010). A simple way to improve anatomical mapping of functional brain imaging. Journal of neuroimaging: official journal of the American Society of Neuroimaging, 20(4), 324333.

West, R. (2011). The Temporal Dynamics of Prospective Memory: A Review of the ERP and Prospective Memory Literature. Neuropsychologia, 49(14), 3795-3800.

West, R., \& Bowry, R. (2005). Effects of aging and working memory demands on 
prospective memory. Psychophysiology, 42(6), 698-712. 


\section{Figures Legends}

Fig.1 Design of the fMRI task. Each block includes instructions, performance prediction, 40 trials (picture + mask), and performance postdiction. Here are illustrated the general procedure and OG-only condition (a), the EBPM (b), and TBPM (c) conditions. Adapted from Gonneaud et al. (2014)

Fig.2 PM-related activity in older adults for EBPM and TBPM conditions. Increased and decreased activity is depicted for EBPM compared to OG-only (left panel), for TBPM compared to OG-only (middle panel) and for EBPM compared to TBPM (right panel). Contrasts are displayed at $p<0.001$ uncorrected, with a minimal cluster size of 69 voxels, and superimposed onto sections of the MNI template using MRIcroN (http://www.mccauslandcenter.sc.edu/mricro/mricron/).

Fig.3 Regions showing more PM-related activity and less PM-related deactivation in older than younger participants. Red clusters represent brain areas more activated during PM by the older group ("PM $>\mathrm{OG}$ in older group" > "PM $>\mathrm{OG}$ in younger group"). Blue clusters depict reduced deactivations in the older group during PM condition (" $\mathrm{OG}>\mathrm{PM}$ in young adults" > "OG $>\mathrm{PM}$ in the older group"). Contrasts are displayed at $p<0.001$ uncorrected, with a minimal cluster size of 69 voxels, and superimposed onto sections of the MNI template using MRIcroN. Mean BOLD signal values (arbitrary units \pm standard error of the mean, SEM) were extracted from all clusters and plotted to illustrate the direction of interactions. $\star \mathrm{p}<0.05$ for the OG-only vs EBPM comparison in young adults; $* \mathrm{p}<0.05$ for the OG-only vs EBPM comparison in older adults. 


\section{Table Legends}

Table 1. Population characteristics.

$\mathrm{SD}=$ Standard Deviation

Table 2. Behavioral performance. Mean percentage of correct answers to PM items, of correct answers to ongoing items (without PM items), and mean reaction time on correct answers to ongoing items (without PM items) according to the group. SD: Standard Deviation 
Table 1

\begin{tabular}{lcc}
\hline & Younger Group & Older Group \\
\hline Age $( \pm S D)$ & $25.15( \pm 5.14)$ & $62.10( \pm 6.30)$ \\
Years of schooling $( \pm S D)$ & $14.45( \pm 2.56)$ & $14.55( \pm 2.70)$ \\
MMSE $( \pm S D)$ & - & $28.70( \pm 0.80)$ \\
MATTIS $( \pm S D)$ & - & $140.85( \pm 2.54)$ \\
Sex ratio (female/male) & $9 / 11$ & $10 / 10$ \\
\hline
\end{tabular}


Table 2

\begin{tabular}{|c|c|c|c|c|c|c|c|}
\hline & & \multicolumn{3}{|c|}{ Young Group } & \multicolumn{3}{|c|}{ Older Group } \\
\hline & & OG-only & EBPM & TBPM & $\begin{array}{l}\text { OG- } \\
\text { only }\end{array}$ & EBPM & TBPM \\
\hline \multirow[t]{2}{*}{ PM items } & Accuracy (\%) & - & 95.17 & 96.17 & - & 86.67 & 76.67 \\
\hline & SD & - & 6.96 & 6.78 & - & 13.33 & 18.03 \\
\hline \multirow{2}{*}{$\begin{array}{l}\text { Ongoing } \\
\text { items }\end{array}$} & Accuracy (\%) & 98.67 & 98.26 & 98.29 & 98.52 & 95.14 & 95.60 \\
\hline & SD & 1.46 & 1.29 & 1.32 & 1.34 & 3.87 & 3.35 \\
\hline \multirow{2}{*}{$\begin{array}{l}\text { Ongoing } \\
\text { items }\end{array}$} & $\begin{array}{l}\text { Reaction Time } \\
\text { (ms) }\end{array}$ & 791.68 & 910.97 & $\begin{array}{c}825.7 \\
6\end{array}$ & 906.70 & $\begin{array}{c}1097.8 \\
4\end{array}$ & $\begin{array}{c}1037.7 \\
2\end{array}$ \\
\hline & SD & 138.77 & 143.29 & $\begin{array}{c}132.0 \\
5\end{array}$ & 90.39 & 127.78 & 127.99 \\
\hline
\end{tabular}




\section{Figure 1}

\section{a. General design}

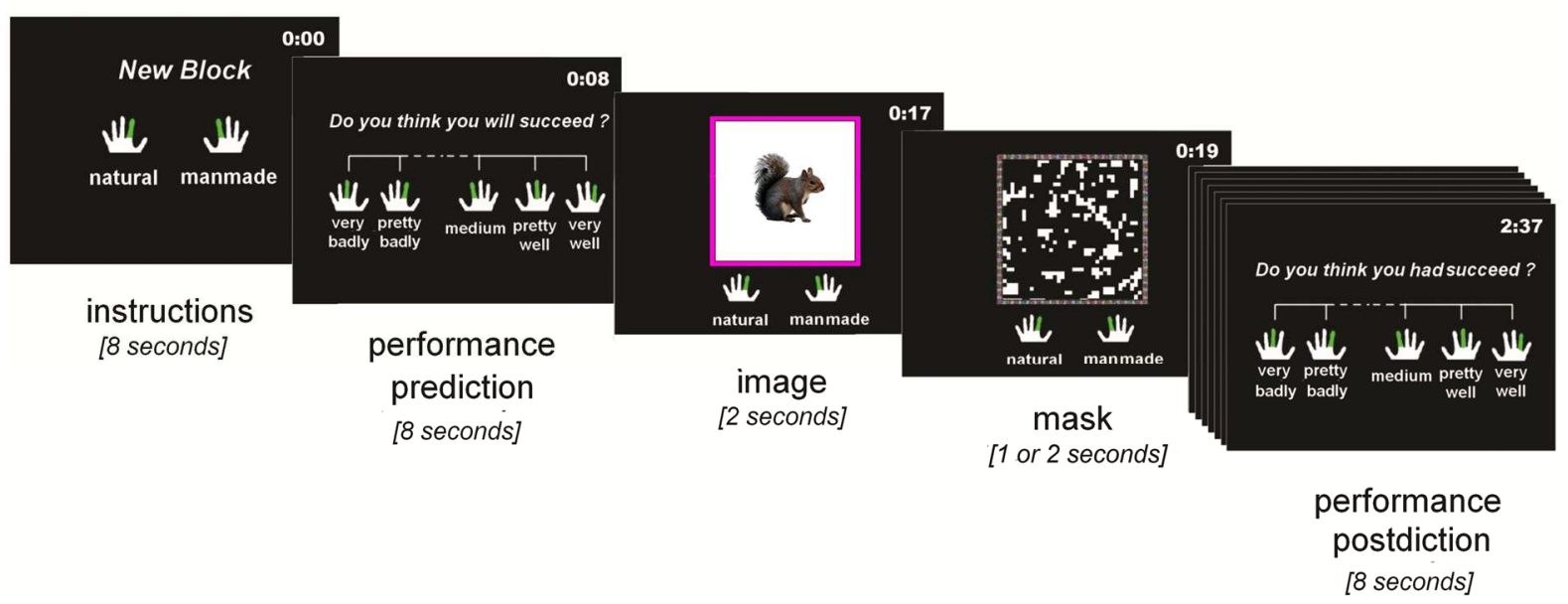

\section{b. EBPM condition}

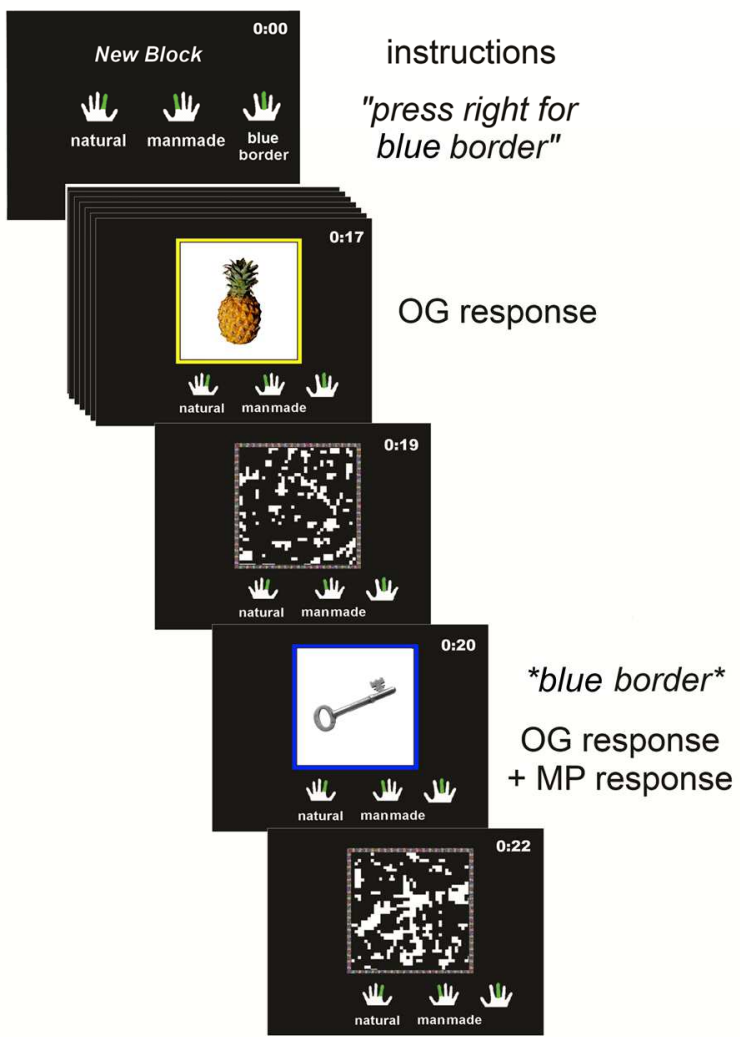

\section{c. TBPM condition}

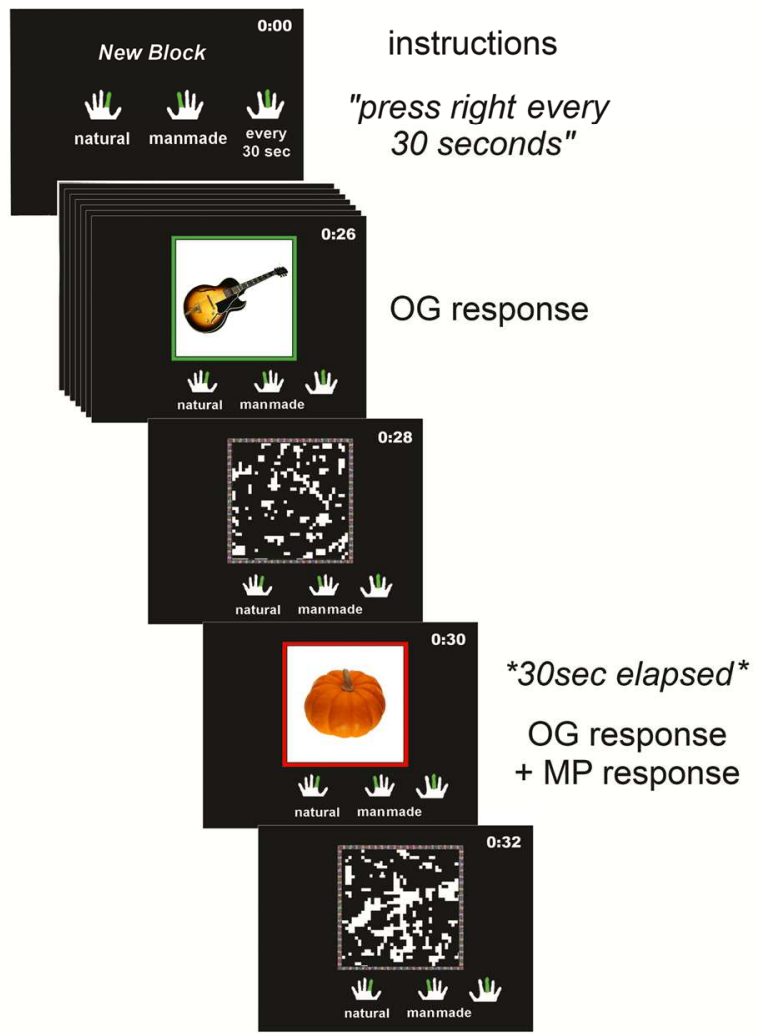


Figure 2

Cerebral substrates of EBPM and TBPM in the older group

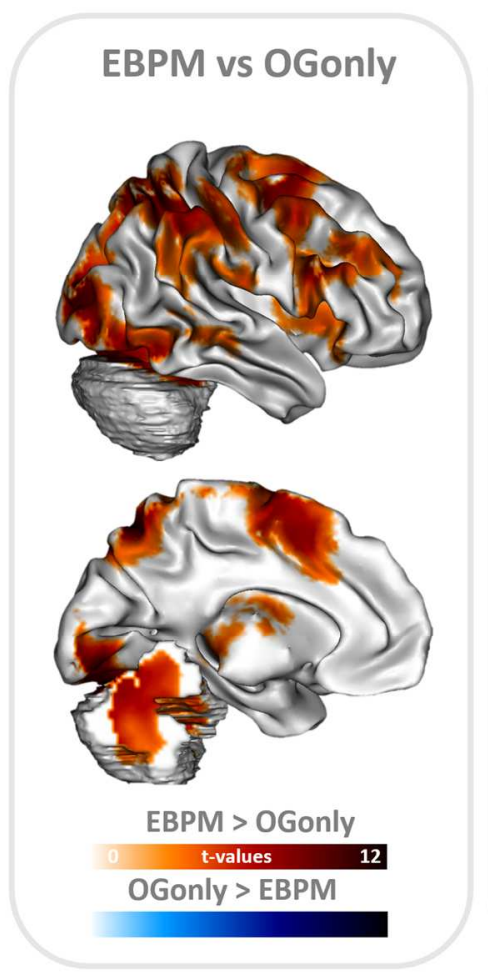

TBPM vs OGonly
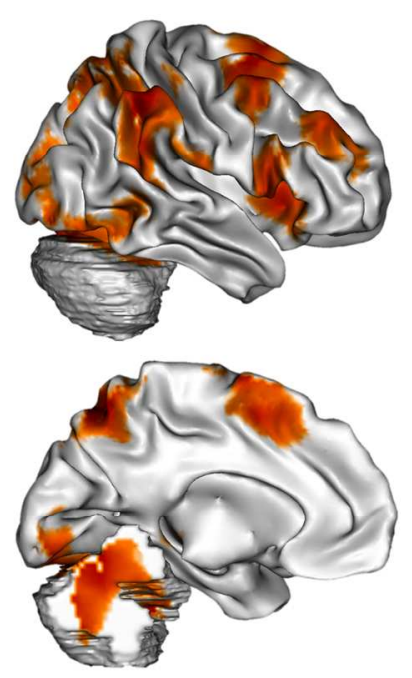

$$
\text { TBPM > OGonly }
$$

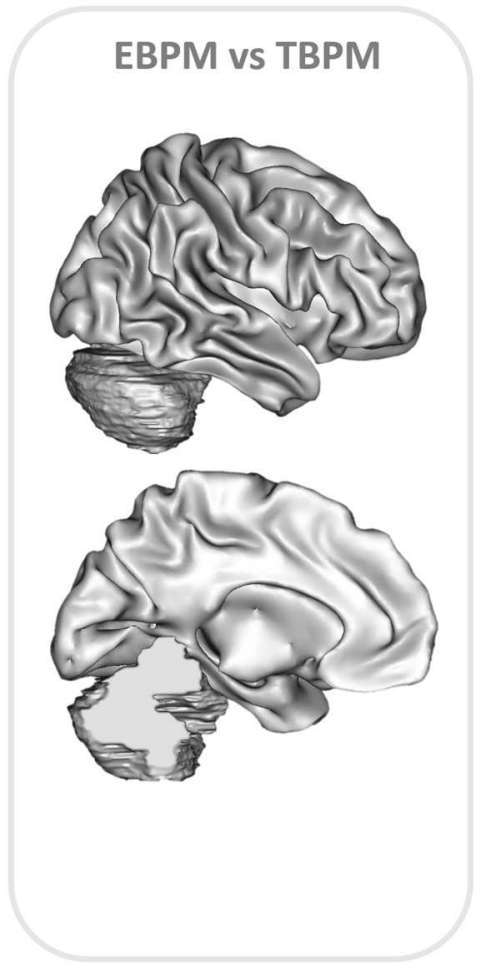


Figure 3

Regions differentially activated by young and older individuals in EBPM
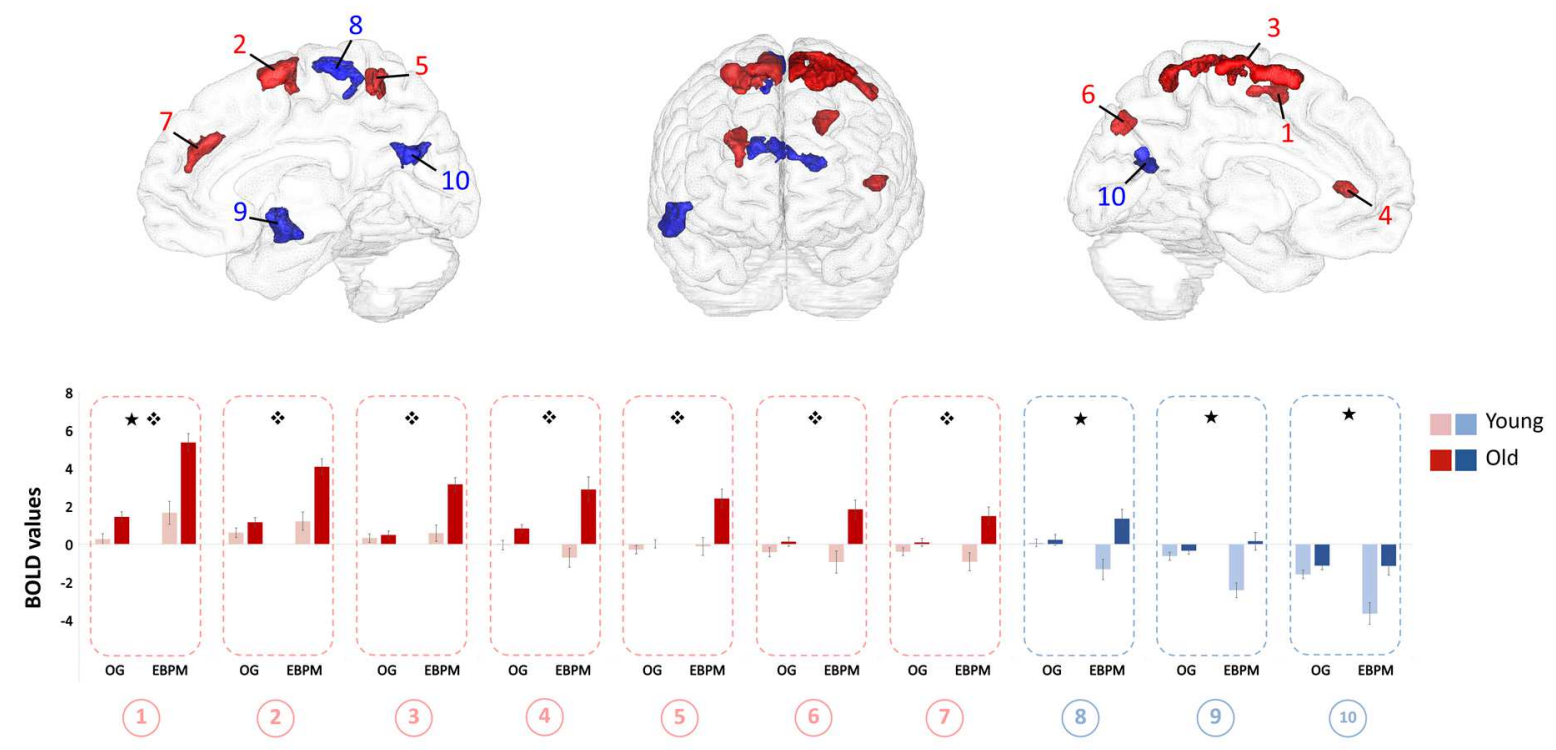

Regions differentially activated by young and older individuals in TBPM
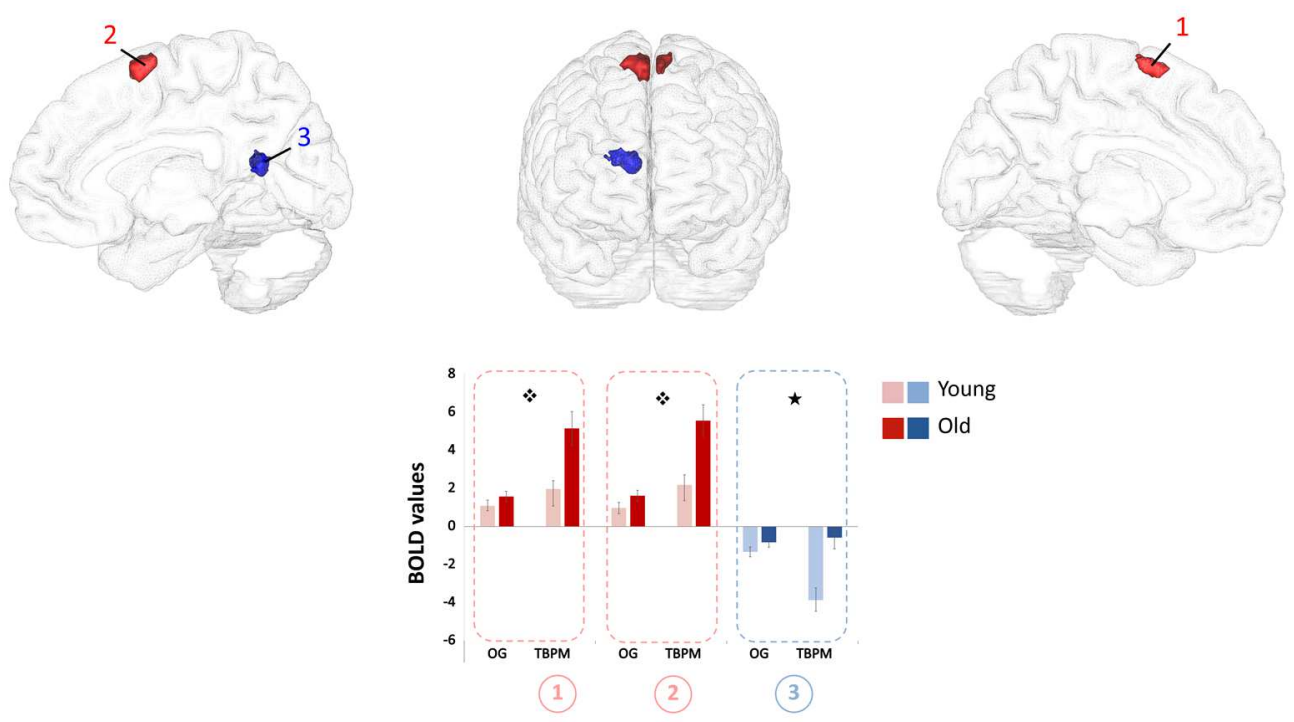